\title{
Noether symmetry of $F(T)$ cosmology with quintessence and phantom scalar
}

\section{fields}

\author{
Mubasher Jamil, $1,2, *$ D. Momeni, $2, \dagger$ and R. Myrzakulov ${ }^{2, \dagger}$ \\ ${ }^{1}$ Center for Advanced Mathematics and Physics (CAMP), \\ National University of Sciences and Technology (NUST), H-12, Islamabad, Pakistan \\ ${ }^{2}$ Eurasian International Center for Theoretical Physics, \\ Eurasian National University, Astana 010008, Kazakhstan
}

\begin{abstract}
In this paper, we investigate the Noether symmetries of $F(T)$ cosmology involving matter and dark energy. In this model, the dark energy is represented by a canonical scalar field with a potential. Two special cases for dark energy are considered including phantom energy and quintessence. We obtain $F(T) \sim T^{3 / 4}$, and the scalar potential $V(\phi) \sim \phi^{2}$ for both models of dark energy and discuss quantum picture of this model. Some astrophysical implications are also discussed.
\end{abstract}

Keywords: Noether symmetries; quintessence; phantom energy; scalar fields; torsion

*Electronic address: mjamil@camp.nust.edu.pk

${ }^{\dagger}$ Electronic address: d.momeni@yahoo.com

${ }^{\ddagger}$ Electronic address: rmyrzakulov@csufresno.edu 


\section{INTRODUCTION}

In the last decade, one of the most active researches in physicist community is investigation of the acceleration of our universe [1], which has confirmed by some observation data such as supernova type Ia [2], baryon acoustic oscillations [3], weak lensing [4] and large scale structure [5]. Finding the theoretical explanation of cosmic acceleration has been one of the central problems of modern cosmology and theoretical physics [6]. Reviews of some recent and old attempts to resolve the issue of dark energy and related problems can be found in [7].

In order to explain the current accelerated expansion without introducing dark energy, one may use a simple generalized version of the so-called teleparallel gravity (TG) [8], namely $F(T)$ theory. It is a generalization of the teleparallel gravity by replacing the so-called torsion scalar $T$ with $F(T)$. TG was originally developed by Einstein in an attempt of unifying gravity and electromagnetism. The field equations for the $F(T)$ gravity are very different from those for $f(R)$ gravity, as they are second order rather than fourth order. $F(T)$ gravity is not locally Lorentz invariant and appear to harbor extra degrees of freedom not present in general relativity [9]. In fact as Li et al [9] pointed out "there are $D-1$ extra degrees of freedom for $F(T)$ gravity in $D$ dimensions, and this implies that the extra degrees of freedom correspond to one massive vector field or one massless vector field with one scalar field." In another recent investigation, Li et al [10] studied the cosmological perturbation and structure formation in $F(T)$ theory and proved that the extra degree of freedom of $F(T)$ gravity decays as one goes to smaller scales, and consequently its effects on scales such as galaxies and galaxies clusters are small. But on large scales, this degree of freedom can produce large deviations from the standard cosmological model, leading to severe constraints on the $F(T)$ gravity models as an explanation to the cosmic acceleration.

Although teleparallel gravity is not an alternative to general relativity (they are dynamically equivalent), but its different formulation allows one to say: gravity is not due to curvature, but to torsion. In other word, using tetrad fields and curvature-less Weitzenbock connection instead of torsion-less Levi-Civita connection in standard general relativity. We should note that one of the main requirement of $F(T)$ gravity is that there exist a class of spin-less connection frames where its

torsion does not vanish [10]. $F(T)$ theory leads to interesting cosmological behavior and its various aspects including thermodynamic laws, phantom crossing and inflation have been examined in the literature [11].

On the other side, we can not ignore the important role of continuous symmetry in the mathematical physics. In particular, the well-known Noether's symmetry theorem is a practical tool in 
theoretical physics which states that any differentiable symmetry of an action of a physical system leads to a corresponding conserved quantity, which so called Noether charge [12]. In the literature, applications of the Noether symmetry in generalized theories of gravity have been studied (see 13 and references therein). In additions, Noether symmetry has been used to investigate non-flat [14] and quantum cosmology [15]. The symmetries of the Lagrangian lead to conserved quantities of the theory, for instance 'total energy' and 'total angular momentum'. If a generic theory does not possesses a conserved charge, it implies that this theory has nothing to do with physical reality. Given the fact that $F(T)$ theory has no symmetry under Lorentz transformation, it will be interesting to know if this theory possesses any other symmetry at all. The application of Noether symmetries helps in selecting viable models of $F(T)$ at a fundamental level. In particular, Wei et al calculated Noether symmetries of $F(T)$ cosmology containing matter and found a power-law solution $F(T) \sim \mu T^{n}$ [13]. Further they showed that if $n>3 / 2$ the expansion of our universe can be accelerated without invoking dark energy. We reconsider their model and invoke a scalar field to produce cosmic acceleration (phantom energy and quintessence as two separate cases) and find $F(T) \sim T^{3 / 4}$. Thus we show that $F(T) \sim T^{3 / 4}$ coupled with a canonical scalar field is equivalent to $F(T) \sim T^{n}, n>3 / 2$.

The plan of our paper is as follows: In section-II, we write down the Lagrangian of our model and related dynamical equations. In section-III, we write down the Noether symmety equations and solve them. In section-IV, we discuss $F(T)$ quantum cosmology. In section-V, we investigate the cosmography and numerical cosmological implications. Finally we conclude the results in section-VI.

\section{OUR MODEL}

Here, we try to consider Noether symmetry in $F(T)$ cosmology in the present of matter and scalar field. The Lagrangian of our model is

$$
\mathcal{S}=\int d^{4} x e\left(\mathcal{L}_{F}+\mathcal{L}_{m}+\mathcal{L}_{\phi}\right)
$$

where $e=\operatorname{det}\left(e_{\mu}^{i}\right)=\sqrt{-g}$, where $e_{i}\left(x^{\mu}\right)$ are related to the metric via $g_{\mu \nu}=\eta_{i j} e_{\mu}^{i} e_{\nu}^{j}$, where all indices run over $0,1,2,3 . \mathcal{L}_{F}, \mathcal{L}_{m}$ and $\mathcal{L}_{\phi}$ represent the Lagrangians for gravity model, energymatter and the scalar field (representing dark energy) respectively. Specifically the total action reads (in chosen units $16 \pi G=\hbar=c=1$ )

$$
\mathcal{S}=2 \pi^{2} \int d t a^{3}\left[F(T)-\rho_{m}+\frac{1}{2} \epsilon \phi_{, \mu} \phi^{, \mu}-V(\phi)\right] .
$$


where $a$ is the scale factor while $H \equiv \dot{a} / a$ is the Hubble parameter. Here $\epsilon=+1,-1$ represent quintessence and phantom (or ghost) dark energies respectively. Although phantom DE is the least desirable candidate of $\mathrm{DE}$ as it violates relativistic energy conditions and leads to future time singularities, we consider it for the sake of completeness of our model since some astrophysical observations support it (see [16] and references therein). The scalar field $\phi$ has the potential energy $V(\phi)$ (to be determined in the later sections) and $\rho_{m}=\rho_{m 0} a^{-3}$ is the energy density of matter with vanishing pressure and $\rho_{m 0}$ is a constant energy density at some initial time.

The Friedmann-Robertson-Walker (FRW) metric representing a spatially flat, homogeneous and isotropic spacetime is given by

$$
d s^{2}=-d t^{2}+a(t)^{2}\left(d x^{2}+d y^{2}+d z^{2}\right)
$$

In FRW cosmological background, the Lagrangian is

$$
\mathcal{S}=2 \pi^{2} \int d t a^{3}\left[F(T)-\lambda\left(T+6 H^{2}\right)-\frac{\rho_{m 0}}{a^{3}}+\frac{1}{2} \epsilon \phi_{, \mu} \phi^{, \mu}-V(\phi)\right] .
$$

where $T=-6 H^{2}$ is the torsion scalar, $\lambda$ is the Lagrange multiplier and can be determined by varying the Lagrangian with respect to torsion scalar $T$, which yields $\lambda=F_{T}$. Integrating by parts in (4), the action converts to

$$
\mathcal{S}=2 \pi^{2} \int d t\left[a^{3}\left(F-T F_{T}\right)-6 F_{T} a \dot{a}^{2}-\rho_{m 0}-a^{3}\left(\frac{1}{2} \epsilon \dot{\phi}^{2}+V(\phi)\right)\right]
$$

and then the point-like Lagrangian reads (ignoring a constant factor $2 \pi^{2}$ )

$$
\mathcal{L}(a, \dot{a}, \phi, \dot{\phi}, T)=a^{3}\left(F-T F_{T}\right)-6 F_{T} a \dot{a}^{2}-\rho_{m 0}-a^{3}\left(\frac{1}{2} \epsilon \dot{\phi}^{2}+V(\phi)\right) .
$$

Moreover for a given dynamical system, the Euler-Lagrange equation is

$$
\frac{d}{d t}\left(\frac{\partial \mathcal{L}}{\partial \dot{q}_{i}}\right)-\frac{\partial \mathcal{L}}{\partial q_{i}}=0
$$

where $q_{i}=a, \phi, T$ are the generalized coordinates of the configuration space $\mathcal{Q}=\{a, \phi, T\}$. Using (6) in (7), we obtain the three equations of motion (corresponding to variations of $\mathcal{L}$ with respect to $T, \phi, a$ respectively)

$$
\begin{gathered}
a^{3} F_{T T}\left(T+6 H^{2}\right)=0, \\
\epsilon(\ddot{\phi}+3 H \dot{\phi})-\frac{\partial V}{\partial \phi}=0, \\
4 \frac{\ddot{a}}{a}\left(F_{T}+2 T F_{T}\right)+4 H^{2}\left(F_{T}-2 T F_{T}\right)+F-T F_{T T}=\rho_{\phi},
\end{gathered}
$$


where

$$
\rho_{\phi} \equiv \frac{1}{2} \epsilon \dot{\phi}^{2}+V(\phi)
$$

Assuming $F_{T T} \neq 0$, from (8) we find

$$
T=-6 H^{2}
$$

which is the torsion scalar for FRW model. Using $\frac{\ddot{a}}{a}=H^{2}+\dot{H}$ and (11) in (10), we find

$$
48 H^{2} F_{T T} \dot{H}-4 F_{T}\left(3 H^{2}+\dot{H}\right)-F=p_{\phi},
$$

is the modified Raychaudhuri equation, where $p_{\phi}=-\rho_{\phi}$ (note that $p=p_{\phi}, p_{m}=0$ ). We also write down the Friedmann equation for our model

$$
12 H^{2} F_{T}+F=\rho_{\phi}+\rho_{m}
$$

\section{NOETHER SYMMETRY ANALYSIS}

The Noether symmetry approach is useful in obtaining exact solution to the given Lagrangian i.e. unknown functions in a given Lagrangian can be determined up to some arbitrary constants.

The Noether symmetry generator is a vector field defined by

$$
\mathbf{X}=\alpha \frac{\partial}{\partial a}+\beta \frac{\partial}{\partial \phi}+\eta \frac{\partial}{\partial T}+\dot{\alpha} \frac{\partial}{\partial \dot{a}}+\dot{\beta} \frac{\partial}{\partial \dot{\phi}}+\dot{\eta} \frac{\partial}{\partial \dot{T}}
$$

where dot represents the total derivative given by

$$
\frac{d}{d t} \equiv \dot{\phi} \frac{\partial}{\partial \phi}+\dot{a} \frac{\partial}{\partial a}+\dot{T} \frac{\partial}{\partial T}
$$

The vector field $\mathbf{X}$ can be thought of as a vector field on $\mathcal{T} \mathcal{Q}=(a, \dot{a}, \phi, \dot{\phi}, T, \dot{T})$ is the related tangent bundle on which $\mathcal{L}$ is defined.

A Noether symmetry $\mathbf{X}$ of a Lagrangian $\mathcal{L}$ exists if the Lie derivative of $\mathcal{L}$ along the vector field $\mathbf{X}$ vanishes i.e.

$$
L_{X} \mathcal{L}=\mathbf{X} \mathcal{L}=\alpha \frac{\partial \mathcal{L}}{\partial a}+\beta \frac{\partial \mathcal{L}}{\partial \phi}+\eta \frac{\partial \mathcal{L}}{\partial T}+\dot{\alpha} \frac{\partial \mathcal{L}}{\partial \dot{a}}+\dot{\beta} \frac{\partial \mathcal{L}}{\partial \dot{\phi}}+\dot{\eta} \frac{\partial \mathcal{L}}{\partial \dot{T}}=0
$$


By requiring the coefficients of $\dot{a}^{2}, \dot{\phi}^{2}, \dot{T}^{2}, \dot{a} \dot{\phi}, \dot{a} \dot{T}$ and $\dot{\phi} \dot{T}$ to be zero in Eq. (16), we find

$$
\begin{aligned}
3 \alpha F-3 \alpha T F_{T}-3 \alpha V(\phi)-\eta a T F_{T T}-\beta a V^{\prime}(\phi) & =0, \\
\alpha F_{T}+\eta a F_{T T}+2 a F_{T} \frac{\partial \alpha}{\partial a} & =0, \\
\frac{3}{2} \alpha+a \frac{\partial \beta}{\partial \phi} & =0, \\
12 F_{T} \frac{\partial \alpha}{\partial \phi}+\epsilon a^{2} \frac{\partial \beta}{\partial a} & =0, \\
12 a F_{T} \frac{\partial \alpha}{\partial T} & =0, \\
\epsilon a^{3} \frac{\partial \beta}{\partial T} & =0 .
\end{aligned}
$$

By assuming $F_{T} \neq 0$, from Eqs. (21) and (22), we conclude

$$
\alpha=\alpha(a, \phi), \quad \beta=\beta(a, \phi) .
$$

Now we must solve the system of equations (17)-(20). The non-trivial solution for this system reads as the following form (Model-I)

$$
\begin{aligned}
F(T) & =\frac{4}{3} c_{1} T^{\frac{3}{4}}+c_{3}, \\
V(\phi) & =c_{4}+c_{5}\left(c_{1} \phi+c_{2}\right)^{2}, \\
\alpha(a, \phi) & =-\frac{2}{3} c_{1} a, \\
\beta(a, \phi, T) & =c_{1} \phi+c_{2}, \\
\eta(a, \phi, T) & =\frac{8}{3} c_{1} T .
\end{aligned}
$$

Here $c_{1} \ldots c_{5}$ are arbitrary constants. It is interesting to note that the form of potential $V$ and torsion function $F$ is the same for both models of dark energy. The quadratic potential (25) has been used to model cosmic inflation including chaotic inflation in super-gravity models [19].

Using (26)-(28), the Noether symmetries are

$$
\mathbf{X}_{1}=-\frac{2}{3} a \frac{\partial}{\partial a}+\frac{8}{3} T \frac{\partial}{\partial T}+\phi \frac{\partial}{\partial \phi}, \quad \mathbf{X}_{2}=\frac{\partial}{\partial T}
$$

The symmetry $\mathbf{X}_{1}$ represents the scaling i.e. the Lagrangian remains invariant under scaling transformation while the second symmetry $\mathbf{X}_{2}$ shows that Lagrangian is invariant under $T$ translation.

These NS generators form a two dimensional closed algebra

$$
\left[\mathbf{X}_{1}, \mathbf{X}_{2}\right]=-\frac{8}{3} \mathbf{X}_{2}
$$


The conjugate momenta for the variables of configuration space $\mathcal{Q}$ can be defined as

$$
\begin{aligned}
& p_{a}=\frac{\partial \mathcal{L}}{\partial \dot{a}}=-12 a \dot{a} F_{T}, \\
& p_{\phi}=\frac{\partial \mathcal{L}}{\partial \dot{\phi}}=\epsilon \dot{a}^{3} \dot{\phi}, \\
& p_{T}=\frac{\partial \mathcal{L}}{\partial \dot{T}}=0 .
\end{aligned}
$$

Notice that $p_{T}=0$ on account of symmetry $\mathbf{X}_{2}$. The Noether charge of the system reads

$$
Q=\alpha p_{a}+\beta p_{\phi}+\eta p_{T}=8 c_{1} a^{2} \dot{a} F_{T}+\left(c_{1} \phi+c_{2}\right) \epsilon \dot{a}^{3} \dot{\phi}
$$

Using (24) in (34), we get

$$
Q=-\frac{32}{36^{1 / 4}} c_{1}^{2}\left(\dot{a} a^{5}\right)^{1 / 2}-a^{3} \epsilon \dot{\phi}\left(c_{1} \phi+c_{2}\right) .
$$

- Remark-1: If $\epsilon=0$, the Noether charge coincides with the results reported in [18] after identifying the parameters $\mu=\frac{4}{3} c_{1}, n=\frac{3}{4}$.

- Remark-2: One obvious solution of the system (17)-(22) is

$$
\alpha=\eta=0, \quad \beta=\text { constant }, \quad V(\phi)=\text { constant }
$$

In this case $a, T$ are cyclic coordinates and we have the following constant charge

$$
Q=\beta p_{\phi}=-\beta \epsilon a^{3} \dot{\phi}
$$

This is the same as the Noether symmetry analysis of purely scalar fields in general relativity [17]. For $Q=0, \phi=$ constant, which corresponds to 'cosmological constant'. But if $Q \neq 0$ then we have $\dot{\phi} \propto a^{-3}$ and the scalar field dilutes with the expansion of the Universe.

- Remark-3: Another interesting solution of the system (17)-(22) is $\epsilon=V(\phi)=0$ which in this case has been discussed in [18]. For the perfect fluids with $\operatorname{EoS} w \neq 0$, the system of Noether symmetry condition is non-integrable.

- Remark-4: We find another interesting solution of a teleparallel gravity with a scalar field (Model-II)

$$
\begin{aligned}
F(T) & =-\frac{3}{16} \epsilon c_{1}^{2} T+c_{4}, \\
V(\phi) & =c_{5} e^{-2 \frac{\phi}{c_{1}}}\left(1+e^{\frac{\phi+c_{2}}{c_{1}}}\right)^{2}, \\
\alpha(a, \phi) & =\frac{2}{3} \sqrt{\frac{-c_{3}}{a}} \sinh \left(\frac{\phi+c_{2}}{c_{1}}\right), \\
\beta(a, \phi) & =\sqrt{\frac{-c_{3}}{a}} \cosh \left(\frac{\phi+c_{2}}{c_{1}}\right), \\
\eta(a, \phi, t) & =\text { arbitrary. }
\end{aligned}
$$


The corresponding Noether charge is given by

$$
Q=\sqrt{\frac{-c_{3}}{a}}\left[-\frac{3}{2} \epsilon c_{1}^{2} a \dot{a} \sinh \left(\frac{\phi+c_{2}}{c_{1}}\right)+\epsilon a^{3} \dot{\phi} \cosh \left(\frac{\phi+c_{2}}{c_{1}}\right)\right] .
$$

\section{IV. $F(T)$ QUANTUM COSMOLOGY}

The Hamiltonian for a given Lagrangian reads

$$
\mathcal{H}=\sum_{i} p_{i} \dot{q}_{i}-\mathcal{L}
$$

Using Eqs. (31)-(33) in (44), we get

$$
\mathcal{H}=-\frac{p_{a}}{24 a F_{T}}-\frac{p_{\phi}^{2}}{\epsilon a^{3}}-a^{3}\left(F-T F_{T}\right)+\rho_{m 0}+a^{3} V(\phi) .
$$

The Hamiltonian equations are

$$
\begin{gathered}
\dot{q}_{i}=\frac{\partial \mathcal{H}}{\partial p_{i}}, \quad \dot{p}_{i}=-\frac{\partial \mathcal{H}}{\partial q_{i}} . \\
\dot{a}=\{a, \mathcal{H}\}, \quad \dot{p}_{a}=\left\{p_{a}, \mathcal{H}\right\}, \\
\dot{T}=\{T, \mathcal{H}\}, \quad \dot{p}_{T}=\left\{p_{T}, \mathcal{H}\right\} \equiv 0, \\
\dot{\phi}=\{\phi, \mathcal{H}\}, \quad \dot{p}_{\phi}=\left\{p_{\phi}, \mathcal{H}\right\} .
\end{gathered}
$$

The Hamiltonian constraint equation $\mathcal{H} \equiv 0$ is equivalent to second Friedmann equation.

The Hamilton-Jacobi equation reads

$$
-\frac{1}{24 a F_{T}} \frac{\partial^{2} S}{\partial a^{2}}-\frac{1}{\epsilon a^{3}} \frac{\partial^{2} S}{\partial \phi^{2}}-a^{3}\left(F-T F_{T}\right)+\rho_{m 0}+a^{3} V(\phi)=0 .
$$

For the quantum picture of our model, we define a wave function $\psi$ and $\frac{\partial}{\partial a_{i}} \rightarrow-\iota \hbar \frac{\partial}{\partial q_{i}}$. Then the wave equation (which is the Hamiltonian constraint) reads:

$$
-\frac{\hbar^{2}}{24 a F_{T}} \frac{\partial^{2} \psi}{\partial a^{2}}-\frac{\hbar^{2}}{\epsilon a^{3}} \frac{\partial^{2} \psi}{\partial \phi^{2}}+U(a, T, \phi) \psi=0
$$

where $U(a, T, \phi)=a^{3}\left(F-T F_{T}\right)-\rho_{m 0}-a^{3} V(\phi)$. Solution of the above wave equation for $F(T)-$ scalar field model is not our main purpose here.

\section{NUMERICAL RESULTS AND COSMOGRAPHY}

In this section, using (24) and (25) in (9) in (10), we obtain the Euler-Lagrange equations:

$$
\begin{aligned}
\epsilon \ddot{\phi}+3 \epsilon H \dot{\phi} & =2 c_{1} c_{5}\left(c_{1} \phi+c_{2}\right), \\
4 \frac{\ddot{a}}{a}\left(F_{T}+2 T F_{T}\right)+4 H^{2}\left(F_{T}-2 T F_{T}\right)+F-T F_{T T} & =\frac{1}{2} \epsilon \dot{\phi}^{2}+c_{4}+c_{5}\left(c_{1} \phi+c_{2}\right)^{2} .
\end{aligned}
$$


We numerically solve (51) and (52) and display our results in the figures 1 to 4 . From figure-1, the e-folding parameter for quintessence increases almost exponentially while for phantom, its stays flat. In figure-2, the quintessence scalar field increases while phantom field oscillates and decay with time. From figure-3, the Hubble parameter decreases from its current value to nearly unity and stays close to zero for phantom while it starts increasing for quintessence. In figure-4, the state parameter for phantom decreases to sub-negative values while for quintessence, it stays near the cosmological constant boundary.

Our model (24) must be checked by observational parameters from cosmographical view, following the methodology presented in [20] we must check the following equations

$$
\begin{aligned}
& f\left(T_{0}\right)=6 H_{0}^{2}\left(\Omega_{m 0}-2\right), \\
& f^{\prime}\left(T_{0}\right)=1 .
\end{aligned}
$$

Explicity we have

$$
\begin{aligned}
& c_{3}=2 H_{0}^{2}\left(3 \Omega_{m 0}-2\right), \\
& c_{1}=-6^{1 / 4} H_{0}^{1 / 2} .
\end{aligned}
$$

Using (55) and (56) in (24) it is possible to find the values of the present value of $F(T)$ and the first derivatives of it using the cosmographic parameters set with a given value of $\Omega_{m 0}$.

\section{A. Reduction of the equations to a single equation for scale factor $a(t)$}

In this section, using (24) and (25) we want to find a single equation for scale factor $a(t)$.

From (51) we get

$$
\epsilon\left(a^{3} \dot{\phi}\right)_{t}=2 c_{1} c_{5} a^{3} \psi
$$

where $\psi=c_{1} \phi+c_{2}$. On the other hand, from (35) we get:

$$
\epsilon a^{3} \dot{\phi}=-\frac{Q+\frac{32}{36^{1 / 4}} c_{1}^{2}\left(\dot{a} a^{5}\right)^{1 / 2}}{\psi}=-\frac{U}{\psi}
$$

where $U=Q+\frac{32}{36^{1 / 4}} c_{1}^{2}\left(\dot{a} a^{5}\right)^{1 / 2}$. Differentation of this equation gives

$$
\epsilon\left(a^{3} \dot{\phi}\right)_{t}=-\frac{\dot{U} \psi-U \dot{\psi}}{\psi^{2}}
$$

So that finally we have

$$
2 c_{1} c_{5} a^{3} \psi=-\frac{\dot{U} \psi-U \dot{\psi}}{\psi^{2}}
$$


From (41) follows

$$
\dot{\psi}=-\frac{c_{1} U}{\epsilon a^{3} \psi}
$$

At least, Eqs. (43) and (44) gives

$$
2 \epsilon c_{1} c_{5} a^{6} \psi^{4}+\epsilon a^{3} \dot{U} \psi^{2}-c_{1} U^{2}=0
$$

Hence we obtain

$$
\psi^{2}= \pm \frac{-\epsilon a^{3} \dot{U} \pm \sqrt{D}}{4 \epsilon c_{1} c_{5} a^{6}}
$$

where

$$
D=a^{6} \dot{U}^{2}+8 \epsilon c_{1}^{2} c_{5} a^{6} U^{2}
$$

For $\psi$ we get the form

$$
\psi=\sqrt{\frac{\sqrt{D} \mp \epsilon a^{3} \dot{U}}{4 \epsilon c_{1} c_{5} a^{6}}}
$$

or

$$
\phi=-c_{2}+\frac{1}{c_{1}} \sqrt{\frac{\sqrt{D} \mp \epsilon a^{3} \dot{U}}{4 \epsilon c_{1} c_{5} a^{6}}}
$$

Now let us rewrite the equation (52) as

$$
[4 \dot{H}(1+2 T)+0.25] c_{1} T^{-0.25}+c_{3}-c_{4}=\frac{2 c_{1} c_{5} U^{2}}{\sqrt{D} \mp \epsilon a^{3} \dot{U}}+\frac{\sqrt{D} \mp \epsilon a^{3} \dot{U}}{4 \epsilon c_{1} a^{6}} .
$$

This equation is very complicated in the form of the $Y(a, \dot{a}, \ddot{a})=0$. We will not solve this equation. From analytical view, there is no simple method for converting this equation to a simpler model. The remaining job is the numerical analysis which we done in previous section.

\section{CONCLUSION}

Noether symmetry analysis is a useful tool to find unknown parameters involved in the Lagrangian. As is observed in the literature, this approach has been used to find explicit forms of $f(R)$ and recently $F(T)$ gravities. In this Letter, we considered the $F(T)$ cosmology with matter and (phantom or quintessence) scalar fields with potential function. Although phantom DE is the least desirable candidate of $\mathrm{DE}$ as it violates relativistic energy conditions and leads to future time singularities, we consider it for the sake of completeness of our model since some astrophysical 
observations support it. Although in literature, one can find numerous forms of $F(T)$ written in an ad hoc way, while the advantage of Noether symmetry is that it helps in calculating a viable form of this arbitrary function. We obtained $F(T) \sim T^{3 / 4}$, and the scalar potential $V(\phi) \sim \phi^{2}$ as a viable candidate of dark energy. In comparison with [18] dealing with $F(T) \sim T^{n}, n>3 / 2$ and matter, our results remain consistent if $n<3 / 2$ and a scalar field is introduced which produces cosmic acceleration. Further we obtained a quadratic potential $V(\phi) \sim \phi^{2}$ for the scalar field. Besides DE, this potential has applications in 'chaotic' inflation model. We also wrote the Schrodinger wave equation for our model and discussed cosmological implications of our model. Our model can be used for the construction of the $F(T)$ Quantum Cosmology.

\section{Acknowledgment}

We would like to thank S.H. Hendi for useful communications and anonymous reviewer for enlightening comments related to this work.

[1] C. L. Bennett, et al., Astrophys. J. Suppl. 148 (2003) 1; D. N. Spergel, et al., Astrophys. J. Suppl. 148 (2003) 175; D. N. Spergel, et al., Astrophys. J. Suppl. 170 (2007) 377.

[2] S. Perlmutter, et al., Astrophys. J. 483 (1997) 565; S. Perlmutter, et al., Nature 391 (1998) 51; S. Perlmutter, et al., Astrophys. J. 517 (1999) 565; A. G. Riess, et al., Astrophys. J. 607 (2004) 665; A. G. Riess, et al., Astrophys. J. 659 (2007) 98.

[3] D. J. Eisentein, et al., Astrophys. J. 633 (2005) 560.

[4] B. Jain and A. Taylor, Phys. Rev. Lett. 91 (2003) 141302.

[5] E. Hawkins, et al., Mon. Not. Roy. Astron. Soc. 346 (2003) 78; M. Tegmark, et al., Phys. Rev. D 69 (2004) 103501; S. Cole, et al., Mon. Not. Roy. Astron. Soc. 362 (2005) 505.

[6] L. Smolin, The Trouble With Physics, (Houghton Mifflin Harcourt 2006).

[7] L. Amendola, S. Tsujikawa, Dark Energy: Theory and Observations (Cambridge University Press, 2010); E. J. Copeland, M. Sami, S. Tsujikawa, Int. J. Mod. Phys. D 15, 1753 (2006); S. Capozziello, M. De Laurentis, arXiv:1108.6266v2 [gr-qc]; S. Nojiri, S.D. Odintsov, Phys. Rept. 505, 59 (2011); T. Padmanabhan, Gen. Rel. Grav. 40, 529 (2008)

[8] A. Einstein, Sitzungsber. Preuss. Akad. Wiss. Phys. Math. Kl., (1928) 217; ibid. (1928) 224; A. Einstein, Math. Ann. 102 (1930) 685; (See A. Unzicker and T. Case, arXiv:physics/0503046 for English translation.) K. Hayashi and T. Shirafuji, Phys. Rev. D 19 (1979) 3524; Addendum-ibid. D 24 (1981) 3312; A. Unzicker and T. Case, arXiv:physics/0503046; R. Aldrovandi and J. G. Pereira, Instituto de Fisica Teorica, UNSEP, Sao Paulo (http://www.ift.unesp.br/gcg/tele.pdf). 
[9] M. Li, R. X. Miao and Y. G. Miao, JHEP 1107 (2011) 108; B. Li, T. P. Sotiriou and J. D. Barrow, Phys. Rev. D 83 (2011) 064035.

[10] T. P. Sotiriou, B. Li, J. D. Barrow, Phys. Rev. D 83 (2011) 104030.

[11] P. Wu and H. W. Yu, Phys. Lett. B 693 (2010) 415; R. Myrzakulov, Eur. Phys. J. C 71 (2011) 1752; K. K. Yerzhanov, S. R. Myrzakul, I. I. Kulnazarov and R. Myrzakulov, arXiv:1006.3879; P. Wu and H. Yu, Phys. Lett. B 692 (2010) 176; P. Y. Tsyba, I. I. Kulnazarov, K. K. Yerzhanov and R. Myrzakulov, Int. J. Theor. Phys. 50 (2011) 1876; G. R. Bengochea, Phys. Lett. B 695 (2011) 405; P. Wu and H. W. Yu, Eur. Phys. J. C 71 (2011) 1552; R. Myrzakulov, arXiv:1008.4486; R. J. Yang, Europhys. Lett. 93 (2011) 60001; K. Bamba, R. Myrzakulov, S. Nojiri, S. D. Odintsov,PhysRevD.85.104036(2012), arXiv:1202.4057; Mubasher Jamil, D. Momeni, Ratbay Myrzakulov, Eur. Phys. J. C72,2075 (2012); Mubasher Jamil, Kuralay Yesmakhanova, Davood Momeni, Ratbay Myrzakulov, Cent. Eur. J. Phys. DOI: 10.2478/s11534-012-0103-2; Y. Zhang, H. Li, Y. Gong and Z. H. Zhu, JCAP 1107 (2011) 015; M. Jamil, D. Momeni, R. Myrzakulov, Eur. Phys. J. C 72, 1959 (2012); K. Bamba, M. Jamil, D. Momeni, R. Myrzakulov, arXiv:1202.6114; H. Wei, X. P. Ma and H. Y. Qi, Phys. Lett. B 703 (2011) 74; C. G. Boehmer, A. Mussa and N. Tamanini, arXiv:1107.4455, K. Bamba, C. Q. Geng, C. C. Lee, L. W. Luo, JCAP 1101 (2011) 021; R. Zheng, Q. G. Huang, JCAP 1103 (2011) 002; R. Ferraro and F. Fiorini, Phys. Lett. B 702 (2011) 75; R. Ferraro , F. Fiorini, Phys. Rev. D 75, 084031 (2007); R. Ferraro, F. Fiorini, Phys. Rev. D 78, 124019 (2008).

[12] E. Noether, Nachr. D. Knig. Gesellsch. D. Wiss. Zu Gttingen, Math-phys. Klasse, (1918) 235257 arXiv:physics/0503066.

[13] H. Wei, X. J. Guo and L. F. Wang, Phys. Lett. B 707, 298 (2012); M. Jamil, F. M. Mahomed, D. Momeni, Phys. Lett. B 702, 315 (2011); I. Hussain, M. Jamil, F. M. Mahomed, strophys. Space Sci. 337, 373 (2012); M. Jamil, S. Ali, D. Momeni, R. Myrzakulov, Eur. Phys. J. C 72, 1998 (2012).

[14] S. Capozziello, R. De Ritis and P. Scudellaro, Nuovo Cim. B 109 (1994) 159.

[15] S. Capozziello, R. De Ritis and P. Scudellaro, Int. J. Mod. Phys. D 3 (1994) 609;S. Capozziello, V. I. Manko, G. Marmo and C. Stornaiolo, Gen. Rel. Grav. 40 (2008) 2627; B. Vakili and F. Khazaie, arXiv:1109.3352 [gr-qc]; B. Vakili, Phys. Lett. B 669 (2008) 206.

[16] M. Jamil, D. Momeni, K. Bamba, Ratbay Myrzakulov, Int. J. Mod. Phys. D 21, (2012) 1250065;Mubasher Jamil , D. Momeni , Chin.Phys.Lett. 28,099801 (2011) ;M. R. Setare,D. Momeni , Int.J.Theor.Phys. 51 ,198(2012); M. R. Setare, , D. Momeni ,Int.J.Mod.Phys. D19,2079(2010) ; M. Jamil, D. Momeni, M. Raza, R. Myrzakulov, Eur. Phys. J. C 72, 1999 (2012); M. Jamil, Y. Myrzakulov, O. Razina, R. Myrzakulov, Astrophys. Space Sci. 336, 315 (2011); M. Jamil, I. Hussain, M. U. Farooq, Astrophys. Space Sci. 335, 339 (2011); M. Jamil, I. Hussain, Int. J. Theor. Phys. 50, 465 (2011); M.R. Setare, M. Jamil, Europhys. Lett. 92, 49003 (2010); A. Sheykhi, M. Jamil, Phys. Lett. B 694, 284 (2011); M. Jamil, M. Akbar, Gen. Rel. Grav. 43, 1061 (2011); M. Jamil, Int. J. Theor. Phys. 49, 144 (2010); M. Jamil, M.A. Rashid, Eur. Phys. J. C 60, 141 (2009); F. De Paolis, M. Jamil, A. Qadir, Int. J. Theor. Phys. 49, 621 (2010). 
[17] Y. Zhang, Y-G. Gong, Z-H. Zhu, Phys. Lett. B 688 (2010) 13.

[18] H. Wei, X.-J. Guo, L.-F. Wang, Phys. Lett. B 707 (2012) 298.

[19] R. Kallosh and A. Linde, JCAP 11 (2010) 011.

[20] S. Capozziello, V. F. Cardone, H. Farajollahi, A. Ravanpak, Phys. Rev. D 84 (2011) 043527. 


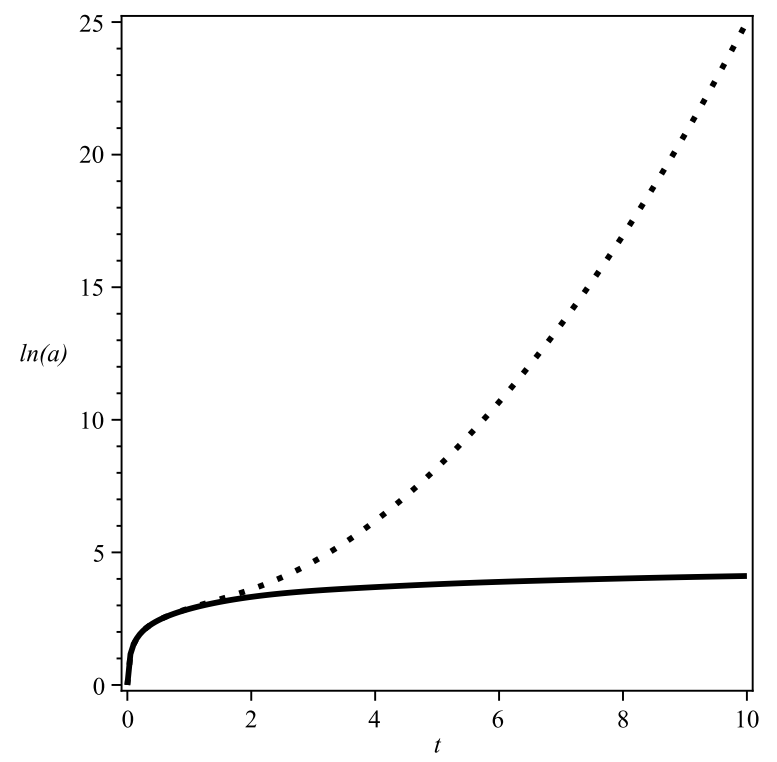

FIG. 1: Graph of $\ln (a)$ vs $t$. The free parameters are chosen as $c_{1}=\frac{3}{4}, \quad c_{2}=c_{4}=0, c_{5}=1$. The inital conditions are $a(0)=\phi(0)=\dot{\phi}(0)=1, \dot{a}(0)=H_{0}$. Here quintessence (phantom) model is shown in dot (line).

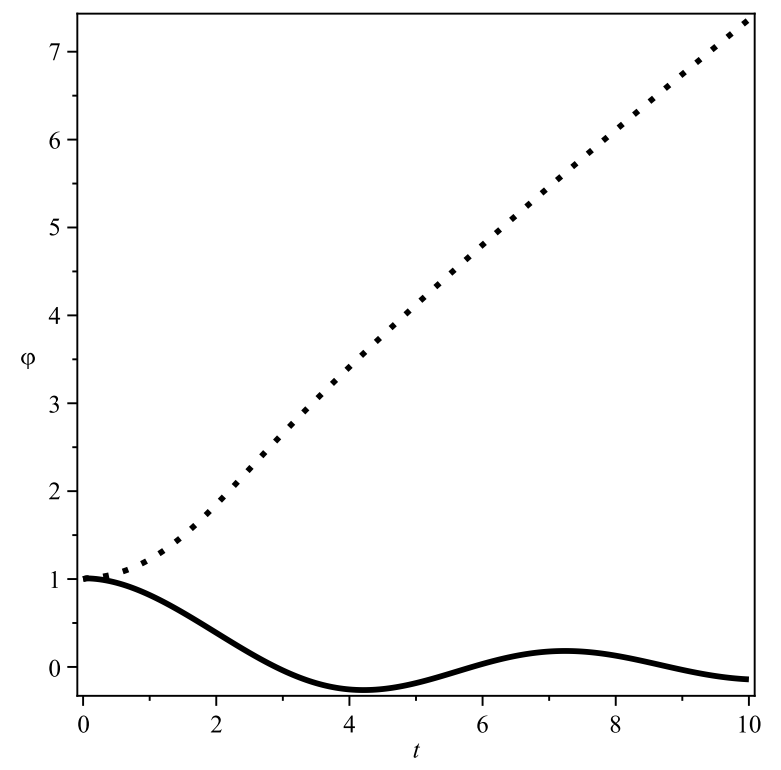

FIG. 2: Graph of $\phi(t)$ vs $t$. The free parameters are chosen as $c_{1}=\frac{3}{4}, \quad c_{2}=c_{4}=0, c_{5}=1$. The inital conditions are $a(0)=\phi(0)=\dot{\phi}(0)=1, \dot{a}(0)=H_{0}$. Here quintessence (phantom) model is shown in dot (line). 


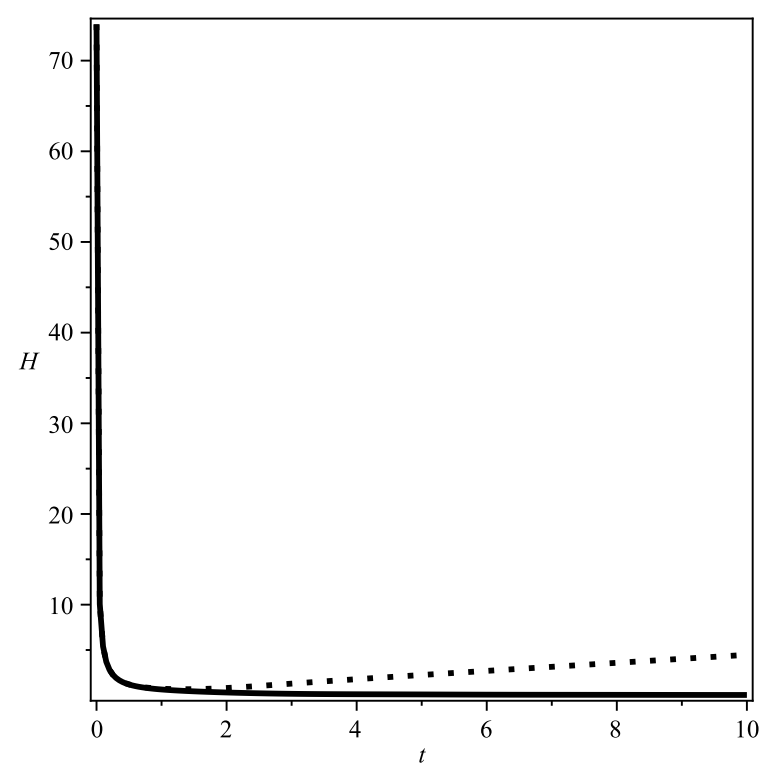

FIG. 3: Graph of $H$ vs $t$. The free parameters are chosen as $c_{1}=\frac{3}{4}, \quad c_{2}=c_{4}=0, c_{5}=1$. The inital conditions are $a(0)=\phi(0)=\dot{\phi}(0)=1, \dot{a}(0)=H_{0}$. Here quintessence (phantom) model is shown in dot (line).

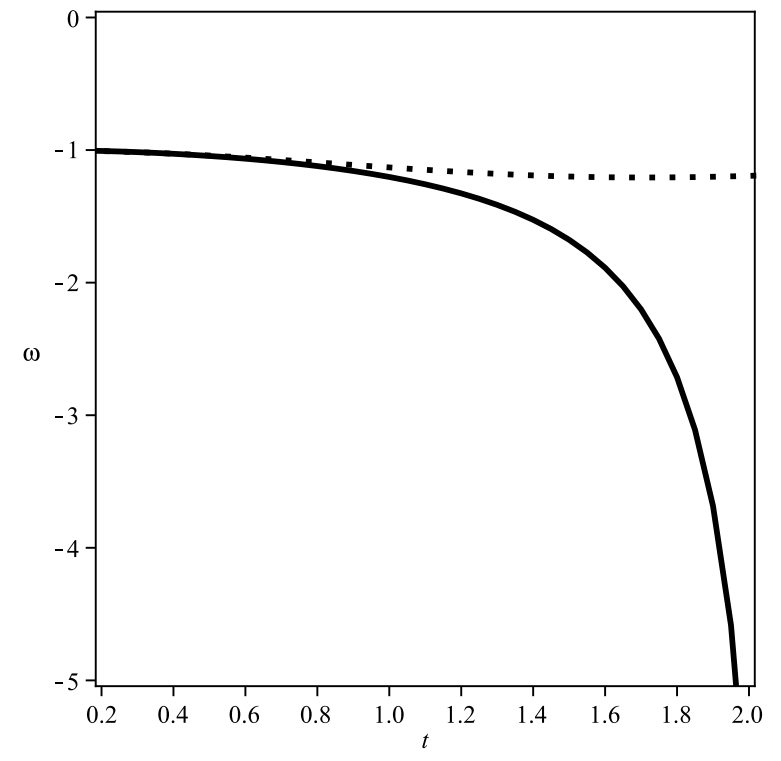

FIG. 4: Graph of $\omega$ vs $t$. The free parameters are chosen as $c_{1}=\frac{3}{4}, c_{2}=c_{4}=0, c_{5}=1$. The initial conditions are $a(0)=\phi(0)=\dot{\phi}(0)=1, \dot{a}(0)=H_{0}$. Here quintessence (phantom) model is shown in dot (line). 